\footnotetext{
${ }^{1}$ Presented at the $16^{\text {th }}$ Annual Congress of the Asia-Pacific Association of Gynecologic Endoscopy and Minimally Invasive Therapy (APAGE) on November 7, 2015, in Beijing, China.

\section{Vaginal Extension Improves Sexual Function in Patients Receiving Laparoscopic} Radical Hysterectomy ${ }^{1}$

\author{
${ }^{1}$ Shanghai OB/GYN Hospital, Fudan University, Shanghai 200011, China \\ ${ }^{2}$ Shanghai Key Laboratory of Female Reproductive Endocrine-Related Diseases, \\ Fudan University, Shanghai, China \\ * These two authors contributed equally to this work.
}

Disclosure and Funding Sources: All authors declare that no conflict of interest exists. This research was supported in part by grants 81270676 (SWG), 81471434 (SWG), 81530040 (SWG) and 81370695 (XSL) from the National Science Foundation of China, and grant 2013ZYJB0019 (XSL) from Shanghai Municipal Commission of Health and Family Planning.

Running title: Vaginal extension improves sexual function

Address correspondence and reprint requests to: Sun-Wei Guo, Ph.D. Shanghai Obstetrics and Gynecology Hospital, Fudan University, 419 Fangxie Road, Shanghai 200011, China (FAX: 86-21-6345-5090; Email: hoxa10@outlook.com); Xishi Liu, M.D., Ph.D., Shanghai Obstetrics and Gynecology Hospital, Fudan University, 419 Fangxie Road, Shanghai 200011, China (FAX: 86-21-6345-5090; Email: $\underline{\text { xsdoc@ @otmail.com) }}$ 


\section{Abstract}

OBJECTIVE: To investigate as whether vaginal extension (VX) following laparoscopic radical hysterectomy (LRH) improve sexual function in patients with early-stage cervical cancer patients.

METHODS: A total of 216 patients with stage Ia1-IIa2 cervical cancer were recruited, 115 of them received LRH concurrently with VX (group VX) and the other 101, LRH only (group C). Demographic, clinicopathological, and peri-operative data were collected. The Female Sexual Function Index (FSFI) questionnaire was administrated before and one year after surgery. Serum estrogen and follicle-stimulating hormone levels were also measured one year after surgery. The total and domain-wise FSFI scores before and after surgery were compared.

RESULTS: Irrespective VX or not, all 6 domains of the FSFI scores in women with early-stage cervical cancer were significantly reduced one year after LRH. VX, however, significantly attenuated this reduction and improved all 6 FSFI domain scores, at the only cost of $<20$ minutes longer operating time. In addition, more ovarian reserve and better pre-operational sexual function also contributed to the attenuation. The ovarian reserve was improved if ovarian preservation procedure was performed during LRH.

CONCLUSIONS: While the sexual function in patients receiving VX procedure does not fully achieve the pre-operational level, the improvement is nonetheless global and significant. Ovarian preservation procedure during LRH may also help improve the sexual function. Therefore, VX and ovarian preservation may be desirable for patients 
47

48

49 Key words: Cervical cancer; Female Sexual Function Index; laparoscopic radical

50 hysterectomy; ovarian preservation; ovarian reserve; sexual function; vaginal

51

extension

52 


\section{Introduction}

Cervical cancer is the second most common female-specific cancer after breast cancer, accounting for $\sim 8 \%$ of both total cancer cases and total cancer deaths in women [1].

While the incidence of new cases and mortality of cervical cancer are both gradually decreasing in the US and many parts of the world [2], the number of newly diagnosed cases appears to be increasing in the coastal regions of China [3, 4]. More disturbingly, most incident cases are in the age group of 15-44 years [4], that is, the most productive and sexually most active period in a woman's life.

Most newly diagnosed cervical cancers are early stage ones, and radical hysterectomy $(\mathrm{RH})$ with pelvic lymphadenectomy has been the standard care. While the prognosis is encouraging, $\mathrm{RH}$ has been well documented to result in considerable changes in anatomy, including vaginal shortening and decreased elasticity of the vaginal wall [5-9]. After parametrial resection, the nerve supply to the vaginal wall could be damaged, yielding inadequate relaxation of the smooth muscle of the vaginal arterio-venous plexus and insufficient vasocongestion and vaginal lubrication when sexually aroused [10]. Oophorectomy also contributes potentially to the pathophysiological mechanism of sexual dysfunction [9]. Consequently, RH results in sexual morbidity, manifested as reduced sexual desire, arousal, and lubrication, and increased dyspareunia, risk of orgasmic disorders, and reduced sexual satisfaction [9,

11]. Unfortunately, the restoration of sexual function or minimizing the risk of sexual 
morbidity for cervical cancer survivors after RH is still an unmet medical need that begs for solution.

To consummate a sexual intercourse and to achieve satisfying sexual experience, a vagina of proper length is one important prerequisite. Yet a shortened vagina following RH is a nagging complaint among cervical cancer survivors [5-7, 12, 13]. To combat this problem, a vaginal extension procedure (VX) has been proposed over half a century ago [14], and VX with posterior peritoneal flap sutured to posterior vaginal wall also has been proposed for patients with cervical cancer after RH [15]. Unfortunately, it is unclear as whether these VX procedures truly improve sexual function or at least attenuate the negative impact resulting from $\mathrm{RH}$.

Ye et al. reported a procedure for VX following RH for patients with FIGO stage Ib1-Ib2 cervical cancer but found no improvement in sex-related dimensions despite extended vaginal length [16]. However, the lack of statistical difference may likely be attributable to its moderate sample sizes. In addition, the psychometric instrument used may not be adequate enough to capture the changes resulting from the VX procedure rendered.

Based on a larger sample size and using the Female Sexual Function Index (FSFI) as a psychometric instrument, Chen et al. reported more recently that peritoneal vaginoplasty following laparoscopic $\mathrm{RH}$ (LRH) improved sexual function in patients 
with early-stage cervical cancer [17]. They report that VX yielded a slight $2.3 \%$, albeit statistically significant, improvement in the total FSFI scores over non-VX group. Given this nearly minute improvement, the statement that VX improves sexual function seems somewhat tenuous at best. After all, a statistically significant event is not necessarily a clinically meaningful one.

Aside from these ambiguities and uncertainties, there is also a question as whether there are other factors, besides vaginal length, are responsible for the sexual function after RH as objectively measured by instruments such as FSFI. Faced with such ambiguities, uncertainties and unknowns, we undertook this study to investigate the effect of VX on sexual function after RH. We hypothesized that VX, in conjunction with ovarian preservation during $\mathrm{RH}$, may improve sexual function in women undergone RH. We compared sexual functions between early-stage cervical patients with and without VX following LRH, assessed before as well as one year after surgery using FSFI based on larger sample sizes. We measured circulating follicle-stimulating hormone (FSH) level, a proxy for ovarian reserve, and attempted to identify factors potentially contributed to the total FSFI scores in addition to vaginal length. We also compared the intra-operative parameters of the two groups.

\section{Materials and Methods}

\section{Patient recruitment and data collection}


One hundred and fifteen patients with FIGO stage Ia1 (with positive margins)-IIa2 cervical cancer who received LRH concurrently with VX procedure using peritoneum at Shanghai OB/GYN Hospital, Fudan University, between January, 2011 and December, 2013, were recruited to this study after informed consent. The criteria for recruitment and inclusion were: 1) younger than 45 years; 2) regularly cycling; 3) sexually active and intended to be so after recovery from surgery; 4) negative history of previous gynecologic operation, and the absence of pelvic adhesion as confirmed during operation ; and 5) negative vaginal margins by intra-operative frozen pathological evaluation. All eligible patients were given detailed explanation regarding the surgical procedure, and possible risks and benefits of VX. It was the patient herself who made the final decision as whether or not to have VX. Informed consent was obtained prior to the operation.

For comparison, an additional 101 stage-matched and married patients (Group C), who received LRH of the same radicality as the VX group, were selected randomly from the pool of 1,191 eligible patients in the same hospital during the same time period were recruited to this study after informed consent. They received the same standard of care except no VX procedure was made during surgery. For all patients, the diagnosis was made by pre-operative pathological biopsies and confirmed by post-operative histology. None of them received any neoadjuvant chemotherapy or radiotherapy prior to the surgery. 
142

143

144

Following the National Comprehensive Cancer Network (NCCN) guidelines [18], when the patient was younger than 45 years and the pre-operative imaging examination showed no ovarian involvement, ovarian preservation was suggested to her before surgery and performed if consented and if gross ovarian biopsy was found to be negative during LRH. In addition, to minimize the impact of postoperative pelvic radiotherapy on ovarian reserve, ovarian transposition was performed who received ovarian preservation. Two patients, one aged 35 and staged Ib2, and the other, aged 39 and staged Ib1, neither with ovarian involvement, still requested bilateral oophorectomy out of concern for possible metastasis, and their wishes were granted.

As with other cervical patients in our hospital, both groups of patients were followed up regularly: if no post-operative adjuvant chemotherapy or radiotherapy was taken, the first follow-up one month after surgery, then every 3 months for the first and second years after surgery, and every 6 months thereafter. If post-operative chemotherapy/radiotherapy was administrated, monthly follow-up was carried for the first 6 months after surgery. Follow-up assessments included pelvic exams, pelvic ultrasonography, vaginal cytology, and serum squamous cell carcinoma antigen test. Computerized tomography or other imaging procedures were also used when deemed necessary.

For both VX and C groups, FSFI questionnaire [19], as a main outcome measurement, was administrated after informed consent within one week before surgery and one 
164

165

year after, as reported previously [20]. FSFI is a well-validated, self-report multiple choice questionnaire for assessing key dimensions or domains of sexual function and quality of life for women. It consists of 6 domains: genital desire, arousal, lubrication, orgasm, sexual satisfaction, and pain. We have used this instrument before [20] and its Chinese version has been validated [21]. In addition, clinical and surgical data were retrieved and recorded, and the information regarding time to resume sexual activity after surgery was also queried and recorded.

Following the NCCN guidelines [18] and the our hospital's guidelines, postoperative adjuvant chemotherapy, radiotherapy or both were recommended to patients deemed to have high risks of metastasis. Following the same guidelines [18], pelvic radiotherapy plus concurrent cisplatin-containing chemotherapy with or without vaginal brachytherapy were advised given the surgical findings of positive pelvic nodes and/or positive surgical margin and/or positive parametrium. Since no patient had positive vaginal mucosal margins, no patient received brachytherapy in this study.

Before and one year after surgery, all recruited patients were interviewed vis-à-vis by a well-trained staff in an isolated room, free from any distraction, and were asked to finish the FSFI questionnaires themselves. The interviewer was blinded to the group assignment. After interview, a gynecologic oncologist evaluated the patient by pelvic examination and measured the vaginal length. After dilating the vagina by a two-bladed speculum, it was examined for any signs of vault dehiscence. To measure 
186

187

vaginal length, a sterile, calibrated glass vaginal stent, wrapped with a condom and properly lubricated, was gently inserted into the vagina down to the vaginal apex and the vaginal length was measured without any stretching [20] and recorded according to the calibration marked on the stent.

All recruited patients were requested for the evaluation of their circulating $\mathrm{FSH}, \mathrm{E}_{2}$, and progesterone $\left(\mathrm{P}_{4}\right)$ levels one year after $\mathrm{RH}$. Due to the absence of menses resulting from the LRH, the time window for measuring the serum FSH levels was determined by the presence or absence of the dominant follicle under abdominal ultrasonography [22]. The circulating $\mathrm{E}_{2}$ and $\mathrm{P}_{4}$ levels were measured simultaneously along with that of FSH. However, 14 (12.2\%) and 24 (23.8\%) patients in the VX and C groups, respectively, did not provide their blood samples. Among them, 2 each from the VX and $\mathrm{C}$ groups died of recurrence and were thus unable to do so, $2 \mathrm{VX}$ patients and $1 \mathrm{C}$ patient were lost to follow-up, $1 \mathrm{C}$ patient had metastasis and had to undergo a second surgery, and $4 \mathrm{VX}$ and $7 \mathrm{C}$ patients did not resume sexual activity. The rest of them simply declined for the measurement.

All patients were followed up to December, 2014, with a minimal follow-up length of 1 year. This study was approved by the Institutional Ethics Review Committee of the Shanghai OB/GYN Hospital. 
LRH and pelvic lymphedenectomy were performed as described in [23], and the uterus was removed through the vaginal canal after RH. Specifically, the modified LRH (Type B, following [24]) was performed on patients with FIGO stage Ia1 cervical cancer coupled with positive margin (4 and 10 in C and VX groups, respectively), and LRH (type C, following [24]) on patients with FIGO stages Ia2 to IIa2 cervical cancer (97 and 105 in C and VX groups, respectively), all in conjunction with pelvic lymph node dissection. No nerve sparing procedure was performed.

When the patient was younger than 45 years and the pre-operative imaging showed no ovarian involvement, ovarian preservation was suggested and performed if gross ovarian biopsy was found to be negative during the LRH. Ovarian transposition was performed to minimize the negative impact of postoperative pelvic radiotherapy. For group $\mathrm{C}$ patients, the vagina cuff was closed with a running locking suture. For VX 222 patients, the VX procedure used in this study was a slightly modified peritoneal vaginoplasty as described by Rangaswamy et al. [25] and has been successfully used by our group for patients with Mayer-Rokitansky-Kuster-Hauser syndrome [20]. Briefly, the uterovesical peritoneum was sutured to the anterior vaginal wall while the rectouterine peritoneum was sutured to the posterior vaginal wall. The 2 horns in the lateral wall were sutured with the homolateral incisal edges of vesical and rectal peritoneum, respectively. Then a purse-string stitch was performed to create the top of the neovagina by taking peritoneum of the bladder and on the surface of the rectum 
4-5 $\mathrm{cm}$ above the vaginal cuff to create an extended neovagina. These sutures were reinforced with non-absorbable Prolene polypropylene surgical thread $0.5 \mathrm{~cm}$ away the previous suturing. The procedure is demonstrated in Supplemental Figure S1. Finally, a $10-\mathrm{cm}$ long and $3-\mathrm{cm}$ wide soft vaginal stent made from gauze, wrapped in two unused condoms. The stent was left in the extended vagina for 48 hours. The perineum was kept sterile and daily vaginal douching was performed.

\section{Statistical analysis}

The comparison of distributions of continuous variables between or among 2 or more groups was made by and remained use of the Wilcoxon rank-sum test and Kruskal-Wallis test, respectively. Spearman's correlation coefficient was used when evaluating correlations between 2 variables. Fisher's exact test was used when comparing the count data between 2 groups. A linear regression analysis was carried out to evaluate whether age at surgery, parity, stage, operating time, amount of blood loss during operation, whether or not ovarian preservation procedure was done during LRH, postoperative serum estrogen levels, FSH, and $\mathrm{E}_{2}$ levels, and vaginal length had any effect on the total or domain-wise FSFI scores evaluated one year after surgery and on the change of the total FSFI score. Similar analyses also were performed for the before-after-RH difference in the total FSFI scores serving as a dependent variable. 
252

253

254

$P$ values of less than 0.05 were considered statistically significant. All computations were made with R 3.2.2 [26] (www.r-project.org).

\section{Results}

\section{Clinicopathological and surgical data}

The clinicopathological and some basic surgical data are presented in Table 1. While patients in the VX group were on average about 2.3 years younger than that of the control group, the two groups were quite comparable with respect to parity, tumor stage, histotypes, complaint of dysmenorrhea and other factors (Table 1). The VX procedure was successful in all patients, and the extended or reconstructed vagina was soft, smooth and elastic. No one in the VX group complained about abnormal discharge or smell. There was no vaginal vault dehiscence in either group.

\section{(Table 1 and Figure 1 about here)}

The added vaginal extension procedure only increased the operating time by an average of 19 min and 24 seconds or a median of 14 minutes (Table 1; Figure 1A). However, the procedure did not increase the amount of blood loss (Table 1; Figure 1B). The two groups also had comparable radicality, rate of ovarian preservation procedure, length of hospital stay, recovery, rate of peri-operational complication, and 
the 1-year overall survival rate (Table 1). In other words, for patients of similar stage and histotype, VX had comparable blood loss, peri-operational complication rate, radicality, and 1-year overall survival rate. The only difference was the additional 19 minutes operating time needed for vaginal extension.

\section{Anatomical and functional outcomes}

One year after surgery, VX patients reported sooner to resume sexual intercourse than that in group C (Table 2). In addition, the VX group had fewer patients who did not have sex due to divorce, death, or other reasons (Table 2). The average vaginal length in the VX group was $8.19( \pm 0.80) \mathrm{cm}$, significantly longer than that in the control group ( $\mathrm{p}<2.2 \times 10^{-16}$; Table 2 and Figure $\left.2 \mathrm{~A}\right)$, which was $5.68( \pm 0.71) \mathrm{cm}$, or on average $2.51 \mathrm{~cm}$ longer. In other words, the improvement in vaginal length due to VX was $44.2 \%$.

Seven patients in the VX group and 11 in the C group did not have FSFI data. Among the 7 patients in the VX group, 1 was due to the death from recurrence, 2 were due to loss to follow-up, 1 was due to divorce, and 3 did not resume sexual activity. In group C, 2 were due to the death from recurrence, 1 was due to loss of follow-up, 1 was due to the metastasis to the left ovary, and 7 did not resume sexual activity. Hence the proportion of women who did not resume their sexual activity is $3 / 111=2.7 \%$ in the VX group and $7 / 97=7.2 \%$ in group $C$, and the difference was not statistically 
significant ( $\mathrm{p}=0.19$, Fisher's exact test).

While there was no significant difference the pre-operational FSFI scores, total or domain-wise, between the two groups, the total FSFI scores were reduced by $38.4 \%$ and $8.8 \%$ in groups C and VX, respectively, after LRH (Table 2; Figure 2B). The VX group had significantly higher post-operative FSFI scores, total or domain-wise, than the control group (Table 2 and Figure 2B). In fact, the average total FSFI scores in VX group was $41.6 \%$ higher than that of group C. Remarkably, both the post-operative total FSFI scores and the change in total FSFI scores before and after surgery correlated positively with the post-operative vaginal length $(r=0.69$, $\mathrm{p}<2.2 \times 10^{-16}$, and $\mathrm{r}=0.58, \mathrm{p}<2.2 \times 10^{-16}$, respectively; Figure $\left.2 \mathrm{C}, \mathrm{D}\right)$

\section{Factors associated with the postoperative FSFI scores}

\section{(Table 2 and Figure 2 about here)}

We also attempted to identify factors associated with the post-operative total FSFI scores. We first attempted this without inclusion of the circulating hormonal levels. By multiple linear regression analysis using age, parity, complaint of dysmenorrhea or not before surgery, post-operative vaginal length, FIGO stage, operating time, amount of blood loss, post-operative adjuvant chemotherapy alone, whether or not had ovarian preservation procedure, radiotherapy alone, and chemoradiotherapy or not as 
covariates, we found that age and the complaint of dysmenorrhea were negatively associated with the post-operative total FSFI score ( $\mathrm{p}=.036$ and $\mathrm{p}=.033$, respectively) while post-operative vaginal length and pre-operative total FSFI scores were positively associated with the score $\left(\mathrm{p}<2.2 \times 10^{-16}\right.$ and $\mathrm{p}=.0002$, respectively; $\left.R^{2}=0.54\right)$. Using the before-after-RH difference of the total FSFI scores yielded essentially the same results except the pre-operative total FSFI scores were negatively associated with the difference $\left(R^{2}=0.72\right)$, indicating that its variance was greater than the covariance of the pre- and post-operative scores.

Next, we added the circulating $\mathrm{FSH}$ and $\mathrm{E}_{2}$ levels into the linear regression. We found that the postoperative circulating FSH levels were negatively associated with the post-operative total FSFI score $\left(\mathrm{p}=1.4 \times 10^{-7}\right)$ while post-operative vaginal length and pre-operative FSFI scores were positively associated with the post-operative score $\left(\mathrm{p}<2.2 \times 10^{-16}\right.$ and $\mathrm{p}=.0016$, respectively; $\left.R^{2}=0.58\right)$. Using the before-after difference of the total FSFI scores resulted in identical results $\left(R^{2}=0.74\right)$. Indeed, the post-operative total FSFI score correlated negatively with the circulating FSH levels $(r=-0.46$, $\mathrm{p}=5.1 \times 10^{-10}$, FSH level was log-transformed; Figure S2) but positively with the circulating $\mathrm{E}_{2}$ levels $\left(\mathrm{r}=0.45, \mathrm{p}=1.2 \times 10^{-9}, \mathrm{E}_{2}\right.$ level was log-transformed to improve normality). Post-operative total FSFI scores also correlated with pre-operative scores $(\mathrm{r}=0.15, \mathrm{p}=.033)$, especially when calculated separately for VX and $\mathrm{C}$ groups (Figure S2CD). Univariate linear regression analysis indicated that vaginal length was responsible for greater share of the variation in the post-operative FSFI scores than 
340

341

342

343

344

the circulating FSH levels $\left(R^{2}=0.47\right.$ vs. $\left.R^{2}=0.21\right)$, and that the pre-operative scores were negligible due to the correlation of the pre/post scores within the same group (Figure S2CD).

Given these results, we further analyzed factors associated with each individual domain scores incorporating age, parity, complaint of dysmenorrhea or not before surgery, post-operative vaginal length, FIGO stage, operating time, amount of blood loss, postoperative adjuvant chemotherapy alone, whether or not had ovarian preservation procedure, whether received the postoperative adjuvant chemotherapy alone, radiotherapy alone, and chemoradiotherapy or not as covariates, and post-operative serum FSH and $\mathrm{E}_{2}$ levels. The results are summarized in Supplemental Table S1. From Table S1, it can be seen that in all 6 domains, vaginal length and serum FSH levels were positively and negatively associated with the scores, respectively, and, except the domains of desire and satisfaction, the baseline (pre-operative) scores were positively associated with the post-operative scores (Table S1). Remarkably, pre-operation complaint of dysmenorrhea was negatively associated with the orgasm and pain domain scores (Table S1). It is noteworthy that in almost all cases and also in the case of total FSFI score, the role of circulating estrogen levels on sexual function was completely eclipsed by the circulating FSH levels.

\section{Determinants of ovarian reserve after $\mathrm{RH}$}


362

363

Since the circulating $\mathrm{E}_{2}$ level is determined by the amount of ovarian function, we wondered which factors were associated with the $E_{2}$ level. Using linear multiple regression analysis incorporating age, parity, whether or not had ovarian preservation procedure, postoperative adjuvant chemotherapy, radiotherapy, and chemoradiotherapy or not, and circulating FSH levels, we found that the serum $\mathrm{E}_{2}$ level was negatively associated with the serum FSH levels alone $\left(\mathrm{p}<2.2 \times 10^{-16}\right.$; $R^{2}=0.73$; Both variables were log-transformed to improve normality). Indeed, the post-operative circulating $\mathrm{E}_{2}$ levels correlated negatively with the circulating FSH levels ( $\mathrm{r}=-0.86, \mathrm{p}<2.2 \times 10^{-16}$; Figure S2B).

Interestingly, using the similar set of covariates but replacing the dependent variable with the serum FSH level, the multiple linear regression analysis indicated the FSH level was negatively associated with the use of ovarian preservation procedure ( $\mathrm{p}=.012$ ) but positively associated with age and the use of adjuvant chemoradiotherapy $\left(\mathrm{p}=3.8 \times 10^{-6}\right.$ and $\mathrm{p}=1.8 \times 10^{-7}$, respectively; $\left.R^{2}=0.31\right)$. That is, the ovarian reserve was impaired by the use of adjuvant chemoradiotherapy, decreased as one aged, and improved if ovarian preservation procedure was performed during $\mathrm{RH}$.

\section{Comparison of changes in FSFI total and domain scores between the two groups}

Using star plots, we plotted the average FSFI domain scores before and after surgery for the control and the VX groups. We found that the C and VX groups are fairly 
384

385

comparable with respect to each FSFI domain (Figure 3), and compared with the full-marked FSFI domain scores (Figure 3), both groups appeared to have substantially reduced score in the "desire" category. However, the score of this category was increased substantially in the VX group but decreased substantially in the C group. On average, scores of all FSFI domains, except the "desire" domain in the VX group, were reduced after surgery, and the reduction was more profound in the C group (Figure 3).

(Figure 3 about here)

\section{Discussion}

Women engage in sexual activity for four general reasons: physical attraction, procreation, emotional bond, and alleviation of insecurity [27]. After RH, physical attraction, emotional bond and insecurity alleviation are likely to become more important reasons for married cervical cancer survivors to engage sexual activity. Unfortunately, the restoration of sexual function for early-stage cervical cancer patients is an unmet medical need that begs for solution. Despite this well-recognized problem $[9,11]$, few studies have been published so far to address this issue.

We have shown in this study that, irrespective VX or not, the sexual function, assessed with either total or domain-wise FSFI scores, in women with early-stage 
cervical cancer is significantly reduced one year after LRH. VX, however, significantly attenuated this reduction in total or domain-wise FSFI scores, at the only cost of slightly longer operating time. In addition, more ovarian reserve and better pre-operational sexual function also contributed to the attenuation of the reduction. Moreover, the ovarian reserve is impaired by the use of postoperative adjuvant chemoradiotherapy, reduced as one aged, and improved if ovarian preservation procedure was performed during $\mathrm{RH}$ as compared with no preservation. While the sexual function in patients receiving VX procedure did not fully achieve the pre-operational level, this is nonetheless very encouraging.

Our study has several strengths. First, we evaluated the patients' sexual function using a well-validated psychometric instrument before and after $\mathrm{RH}$, in addition to our larger sample sizes. Second, we supplemented our analysis with a proxy of ovarian reserve. Lastly, we analyzed several factors that may potentially impact on sexual function.

Our study also has limitations. Firstly, the fact that the average age of the VX group is about 2 years younger than that of the $\mathrm{C}$ group is probably due to somewhat directional pre-operative consultation with patients. This highlights the limitations of this study, i.e. the non-random nature of group assignment. Upon recognition of this deficiency, we remedied our study by the use of blind evaluation and multiple linear regression, which minimized the bias of non-random assignment. Secondly, the 
comparable baseline FSFI scores in all domains between the two groups also suggest that the two groups are comparable. Second, we used serum FSH levels as a proxy for ovarian reserve, which are not as good as antral follicle count or anti-Müllerian hormone levels $[28,29]$. Thirdly, we did not enumerate the patients' reasons for not having sexual intercourse one year after surgery, and this could potentially introduce an important bias in our results. While some possible reasons, such as the fear for contracting HPV again through sexual transmission and thus increasing the risk of metastasis and/or recurrence, could be gleaned from conversations with the patients, we note that these reasons were spoken ones and hence should be viewed, at best, as "exterior" reasons. There may well be some other unspoken and ulterior reasons, e.g. the anger towards their partners for transmitting HPV to them and thus refusing to engage any sexual activity or even intimacy. However, to get to the bottom of the truth may require efforts well beyond our professional boundary. Fortunately, the number of people who did not resume sex one year after surgery was small in both groups, and the difference did not reach statistical significance. Therefore, it is unlikely that omission of these patients would entail any bias. Lastly, since the sexual function of cervical cancer survivors encompasses not only hardware but also software which may include, but not limited to, the extent of intimacy and the performance of their partners, our study, like many others, provides only a glimpse at the sexual function after RH. Much more research is warranted to identify all factors that affect the function in these patients. 
450

451

452

453

454

455

456

457

458

459

460

461

462

463

464

465

466

467

468

469

470

471

Voluminous research has shown that RH impacts negatively on sexual function in patients with early-stage cervical cancer $[5-7,10,16]$. Thus, our results that the total and domain-wise FSFI scores are significantly decreased after surgery---irrespective VX---are consistent with the published data. As we have shown, scores of all 6 FSFI domains were significantly reduced one year after RH (Table 2 and Figure 2), indicating that RH impacted negatively on all 6 aspects of female sexual functioning. Indeed, RH yields considerable changes in anatomy, including vaginal shortening and decreased elasticity of the vaginal wall [5-9].

In contrast to the study reported by Ye et al. [16], we found that VX following RH significantly attenuated the decrease in sexual function due to $\mathrm{RH}$ and improved all 6 domains of FSFI. In particular, the domain of sexual desire seems to be much improved after VX as compared with that before surgery. Moderate sample sizes ( $n=31$ and $n=28$ in the two arms) that they used could have conferred inadequate statistical power. The psychometric instrument used also may not be adequate enough to capture the changes resulting from the VX procedure.

Our findings also contrast sharply with that reported recently by Chen et al. [17]. As with the study by Ye et al. [16], this study did not have the pre-operative baseline measurement, either. Consequently, it is difficult to gauge as whether the patients with and without VX were genuinely comparable before RH, even though demographically and clinicopathologically they were. In addition, given the reported average vaginal 
472

473

length in the VX group that was over twice as long as that of the non-VX group but merely $2.3 \%$, albeit statistically significant, improvement in the total FSFI scores over the non-VX group [17], the statement that VX improves sexual function may sound somewhat anti-climax and the VX may be construed as an overkill. In contrast, the average vaginal length in the VX group was $44.2 \%$ longer than group C, which seems to be more in line with the average improvement in total FSFI scores in VX group by $41.6 \%$.

As per the requirements of RH for early-stage cervical cancer, approximately $3-\mathrm{cm}$ vaginal wall and parametrium are resected, which inevitably reduces the vaginal length and damages the peripheral nerves and small blood vessels around the uterus. The end results are a substantially shortened vagina, reduced elasticity, loss of sensation, and reduced vaginal lubrication. Given almost surely sexual dysfunction in women following $\mathrm{RH}$, it is somewhat remarkable that the VX procedure can significantly alleviate such deterioration, especially at the cost of slightly increased operating time without any compromise in radicality, amount of blood loss, the intra-operative complication rate, or recovery. The VX procedure seems to be able to remedy the problem of reduced vaginal length, attenuating the negative impact of RH on sexual function. In fact, VX seems to improve the sexual function of cervical cancer survivors globally, possibly because their extended vagina may help overcome the sense of inadequacy and reclaim their womanhood. Since the procedure does not 
494

495

496

497

498

499

500

address other complications resulting from $\mathrm{RH}$, such as damage to the nerve and small blood vessels, it is somewhat not surprising that the sexual function for women receiving $\mathrm{RH}$ is not completely recovered even after VX.

In fact, our results demonstrated that more ovarian reserve also is an important factor for all domains of FSFI. Indeed, diminished circulating estrogen levels are responsible for vaginal thinning, tightening, dryness, or even atrophy, all of which may negatively impact on all domains of the FSFI, as seen in Table S1. Yet the sole determinant of the serum estrogen levels is the ovarian reserve, which can be evaluated by the basal serum FSH level [29]. Further analysis indicates that the condition of ovarian reserve in patients following $\mathrm{RH}$ depends on whether postoperative adjuvant chemoradiotherapy is administrated or not, whether or not ovarian preservation procedure is performed during surgery, and, not surprisingly, age. This is consistent with the common sense that age is a critical determinant of the ovarian reserve and that the performance of the ovarian preservation procedure can preserve the ovarian reserve. It is also agrees with the published data that chemotherapy causes depletion of the primordial follicle pool and thus ovarian reserve [30] and radiotherapy increases the risk of sexual morbidity following RH [31, 32]. More importantly, our results indicate that the condition of the ovarian reserve one year after $\mathrm{RH}$ is determined, roughly equally, by three factors: age, whether ovarian preservation procedure is employed or not, and whether chemoradiotherapy is administrated or not. The use of ovarian preservation procedure during LRH can ultimately help improve 
sexual function through better preserving ovarian reserve.

Our results also showed that postoperative FSFI scores are correlated positively with pre-operative ones (Figure S2). This may be attributable to the notion that, everything being equal, women who had higher libido, more erotic fantasies, or simply more intimate relationship with their spouses before surgery may have continued to do so after, thus the positive correlation between pre- and post-operative FSFI scores.

While pre-operative sexual function, post-operative vaginal length and ovarian reserve were found to be associated with the post-operative total FSFI scores, we note that these three factors combined could only explain about $60 \%$ of the variation in the post-operative FSFI scores, as evidenced by the $R^{2}$ of the regression model. In other words, there are still other factors, suspected or yet to be identified, that are responsible for the sexual function in cervical cancer survivors following RH. From an interventional standpoint, perhaps a combination of VX, ovarian preservation, the use of chemoradiotherapy that has less side-effects and causes less damage to the ovaries, the use of nerve-sparing procedure during laparoscopic or conventional RH [33], and counseling and rehabilitation before and after surgery [32] may help to restore at least a bigger portion of the patients' pre-operation sexual functional level. In particular, a multicenter comparative study and a randomized clinical trial have shown that nerve-sparing RH confers better quality of life, sexual function [34], and bladder function without compromise in long-term survival [35]. Therefore, aside 
538

539

540

541

542

543

544

545

546

547

548

549

550

551

552

553

554

555

556

557

558

559

from vaginal extension and ovarian preservation, there are additional ways to improve sexual functions in cervical cancer survivors undergone RH, as depicted in Figure 4.

\section{(Figure 4 about here)}

To conclude, our study provides evidence that VX significantly attenuated the decrease in sexual function after RH, at the only cost of less than 20 minutes longer operating time. While VX did not fully recover the sexual function following RH, it, combined with the ovarian preservation procedure, improves total and all domains of female sexual functions. Consequently, vaginal extension, along with ovarian preservation procedure, may be desirable for patients with early-stage cervical cancer undergoing RH.

\section{Acknowledgement}

This work was supported in part by grants 81270676 (SWG), 81471434 (SWG), 81530040 (SWG) and 81370695 (XSL) from the National Science Foundation of China, and grant 2013ZYJB0019 (XSL) from Shanghai Municipal Commission of Health and Family Planning. 


\section{References}

[1] Organization. WH. World Cancer Report 2014.2014.

[2] Global Burden of Disease Cancer C, Fitzmaurice C, Dicker D, Pain A, Hamavid H, Moradi-Lakeh M, et al. The Global Burden of Cancer 2013. JAMA Oncol. 2015;1:505-27.

[3] Aminimoghaddam S, Shahrabi-Farahani M, Mohajeri-Tehrani M, Amiri P, Fereidooni F, Larijani B, et al. Epistatic interaction between adiponectin and survivin gene polymorphisms in endometrial carcinoma. Pathol Res Pract. 2015;211:293-7.

[4] Du LB, Li HZ, Wang XH, Zhu C, Liu QM, Li QL, et al. Analysis of cancer incidence in Zhejiang cancer registry in China during 2000 to 2009. Asian Pac J Cancer Prev. 2014;15:5839-43.

[5] Bergmark K, Avall-Lundqvist E, Dickman PW, Henningsohn L, Steineck G. Vaginal changes and sexuality in women with a history of cervical cancer. $\mathrm{N}$ Engl J Med. 1999;340:1383-9.

[6] Grumann M, Robertson R, Hacker NF, Sommer G. Sexual functioning in patients following radical hysterectomy for stage IB cancer of the cervix. Int J Gynecol Cancer. 2001;11:372-80.

[7] Donovan KA, Taliaferro LA, Alvarez EM, Jacobsen PB, Roetzheim RG, Wenham RM. Sexual health in women treated for cervical cancer: characteristics and correlates. Gynecol Oncol. 2007;104:428-34.

[8] Jensen PT, Groenvold M, Klee MC, Thranov I, Petersen MA, Machin D. 
582

583

584

585

586

587

588

589

590

591

592

593

594

595

596

597

598

599

600

601

602

603

Early-stage cervical carcinoma, radical hysterectomy, and sexual function. A longitudinal study. Cancer. 2004;100:97-106.

[9] Vrzackova P, Weiss P, Cibula D. Sexual morbidity following radical hysterectomy for cervical cancer. Expert Rev Anticancer Ther. 2010;10:1037-42.

[10] Gallo-Silver L. The sexual rehabilitation of persons with cancer. Cancer Pract. 2000;8:10-5.

[11] Lonnee-Hoffmann R, Pinas I. Effects of Hysterectomy on Sexual Function. Curr Sex Health Rep. 2014;6:244-51.

[12] Burns M, Costello J, Ryan-Woolley B, Davidson S. Assessing the impact of late treatment effects in cervical cancer: an exploratory study of women's sexuality. Eur $\mathbf{J}$ Cancer Care (Engl). 2007;16:364-72.

[13] Park SY, Bae DS, Nam JH, Park CT, Cho CH, Lee JM, et al. Quality of life and sexual problems in disease-free survivors of cervical cancer compared with the general population. Cancer. 2007;110:2716-25.

[14] Symmonds RE, Pratt JH. Prevention of fistulas and lymphocysts in radical hysterectomy. Preliminary report of a new technic. Obstet Gynecol. 1961;17:57-64.

[15] Saito M, Kumasaka T, Kato K, Yazawa K. Vaginal repair in the radical operation for cervical carcinoma. Acta Obstet Gynecol Scand. 1976;55:151-4.

[16] Ye S, Yang J, Cao D, Zhu L, Lang J, Chuang LT, et al. Quality of life and sexual function of patients following radical hysterectomy and vaginal extension. J Sex Med. $2014 ; 11: 1334-42$.

[17] Chen SQ, Kong LZ, Jiang HY, Fan L, Chen J, Yao SZ. Early cervical cancer 
604

605

606

607

608

609

610

611

612

613

614

615

616

617

618

619

620

621

622

623

624

625

impact of peritoneal vaginoplasty combined with laparoscopic radical hysterectomy improved sexual function. Int J Gynecol Cancer. 2015;25:526-32.

[18] Koh WJ, Greer BE, Abu-Rustum NR, Apte SM, Campos SM, Cho KR, et al.

Cervical Cancer, Version 2.2015. J Natl Compr Canc Netw. 2015;13:395-404; quiz

[19] Rosen R, Brown C, Heiman J, Leiblum S, Meston C, Shabsigh R, et al. The

Female Sexual Function Index (FSFI): a multidimensional self-report instrument for the assessment of female sexual function. J Sex Marital Ther. 2000;26:191-208.

[20] Liu X, Liu M, Hua K, Li B, Guo SW. Sexuality after laparoscopic peritoneal vaginoplasty in women with Mayer-Rokitansky-Kuster-Hauser syndrome. J Minim Invasive Gynecol. 2009;16:720-9.

[21] Sun X, Li C, Jin L, Fan Y, Wang D. Development and validation of Chinese version of female sexual function index in a Chinese population-a pilot study. J Sex Med. 2011;8:1101-11.

[22] Matijevic R, Grgic O. Predictive values of ultrasound monitoring of the menstrual cycle. Curr Opin Obstet Gynecol. 2005;17:405-10.

[23] Nezhat CR, Burrell MO, Nezhat FR, Benigno BB, Welander CE. Laparoscopic radical hysterectomy with paraaortic and pelvic node dissection. Am J Obstet Gynecol. 1992;166:864-5.

[24] Querleu D, Morrow CP. Classification of radical hysterectomy. Lancet Oncol. 2008;9:297-303.

[25] Rangaswamy M, Machado NO, Kaur S, Machado L. Laparoscopic vaginoplasty: using a sliding peritoneal flap for correction of complete vaginal agenesis. Eur $\mathbf{J}$ 
626

627

Obstet Gynecol Reprod Biol. 2001;98:244-8.

[26] Inhaka R, Gentleman RR. R: a language for data analysis and graphics. J comput Graph Statist. 1996;5:1923-7.

[27] Meston CM, Buss DM. Why women have sex: Women reveal the truth about their sex lives, from adventure to revenge (and everything from between). . Reprint edition ed: St. Martin's Griffin; 2010.

[28] Anderson RA, Themmen AP, Al-Qahtani A, Groome NP, Cameron DA. The effects of chemotherapy and long-term gonadotrophin suppression on the ovarian reserve in premenopausal women with breast cancer. Hum Reprod. 2006;21:2583-92.

[29] Committee on Gynecologic P. Committee opinion no. 618: Ovarian reserve testing. Obstet Gynecol. 2015;125:268-73.

[30] Sonmezer M, Oktay K. Fertility preservation in female patients. Hum Reprod Update. 2004;10:251-66.

[31] Pieterse QD, Kenter GG, Maas CP, de Kroon CD, Creutzberg CL, Trimbos JB, et al. Self-reported sexual, bowel and bladder function in cervical cancer patients following different treatment modalities: longitudinal prospective cohort study. Int J Gynecol Cancer. 2013;23:1717-25.

[32] Harding Y, Ooyama T, Nakamoto T, Wakayama A, Kudaka W, Inamine M, et al. Radiotherapy- or radical surgery-induced female sexual morbidity in stages IB and II cervical cancer. Int J Gynecol Cancer. 2014;24:800-5.

[33] Bogani G, Serati M, Nappi R, Cromi A, di Naro E, Ghezzi F. Nerve-sparing approach reduces sexual dysfunction in patients undergoing laparoscopic radical 
648

649

650

651

652

653

654

655

hysterectomy. J Sex Med. 2014;11:3012-20.

[34] Ceccaroni M, Roviglione G, Spagnolo E, Casadio P, Clarizia R, Peiretti M, et al.

Pelvic dysfunctions and quality of life after nerve-sparing radical hysterectomy: a multicenter comparative study. Anticancer Res. 2012;32:581-8.

[35] Roh JW, Lee DO, Suh DH, Lim MC, Seo SS, Chung J, et al. Efficacy and oncologic safety of nerve-sparing radical hysterectomy for cervical cancer: a randomized controlled trial. J Gynecol Oncol. 2015;26:90-9. 
656

657

658

659

660

661

662

663

664

665

666

667

668

669

670

671

672

673

674

675

676

677

\section{Figure legend}

Figure 1. (A) Boxplot showing the distribution of operating time (in minutes)

between patients with and without vaginal extension following laparoscopic radical hysterectomy. The number shown in the figure is the p-value of the difference. (B)

Boxplot showing the distribution of the amount of blood loss (in $\mathrm{mL}$ ) between

patients with and without vaginal extension following laparoscopic radical hysterectomy. NS statistically not significant, i.e. $\mathrm{p}>0.05$.

Figure 2. (A) Boxplot showing the vaginal length (in $\mathrm{cm}$ ) between patients with and without vaginal extension following laparoscopic radical hysterectomy; The number shown in the figure is the p-value of the difference. (B) Boxplot showing the distribution of the total FSFI scores between patients with and without vaginal extension following laparoscopic radical hysterectomy before and one year after the surgery. C: Control, i.e. no vaginal extension; VX: vaginal extension; pre-op:

Pre-operation; post-op: one year post-operation; ***: Statistically highly significant, i.e. $\mathrm{p}<0.001$; NS: statistically not significant, i.e. $\mathrm{p}>0.05$. (C) Scatter plot showing the relationship between vaginal length and the total FSFI scores, both evaluated one year after surgery. (D) Scatter plot showing the relationship between post-operational vaginal length and the before-after change of total FSFI scores. The dots in blue are those patients with VX while those in red are patients without. The numbers shown in (C) and (D) are Pearson's correlation coefficients and their statistical significance. $* * *$ : 
678

Statistically highly significant, i.e. $\mathrm{p}<0.001$. In both (C) and (D), the black dashed line

679 represents the regression line. The green dotted line in (D) indicates no change.

680

681

682

683

684

685

686

687

688

689

690

691

692

Figure 3. Comparison of the average FSFI domain scores in patients with and without vaginal extension following laparoscopic radical hysterectomy before and one year after the surgery.

Figure 4. Diagram showing possible ways to affect/improve the sexual function for women with early-stage cervical cancer undergoing radical hysterectomy. The greenish arrows indicate promotional/facilitory/improving effects, while the red one indicates inhibitory effects. The question mark indicates uncertainty or unknown. The rectangles with rounded corners are factors that can be potentially intervened during laparoscopic radical hysterectomy and after. 

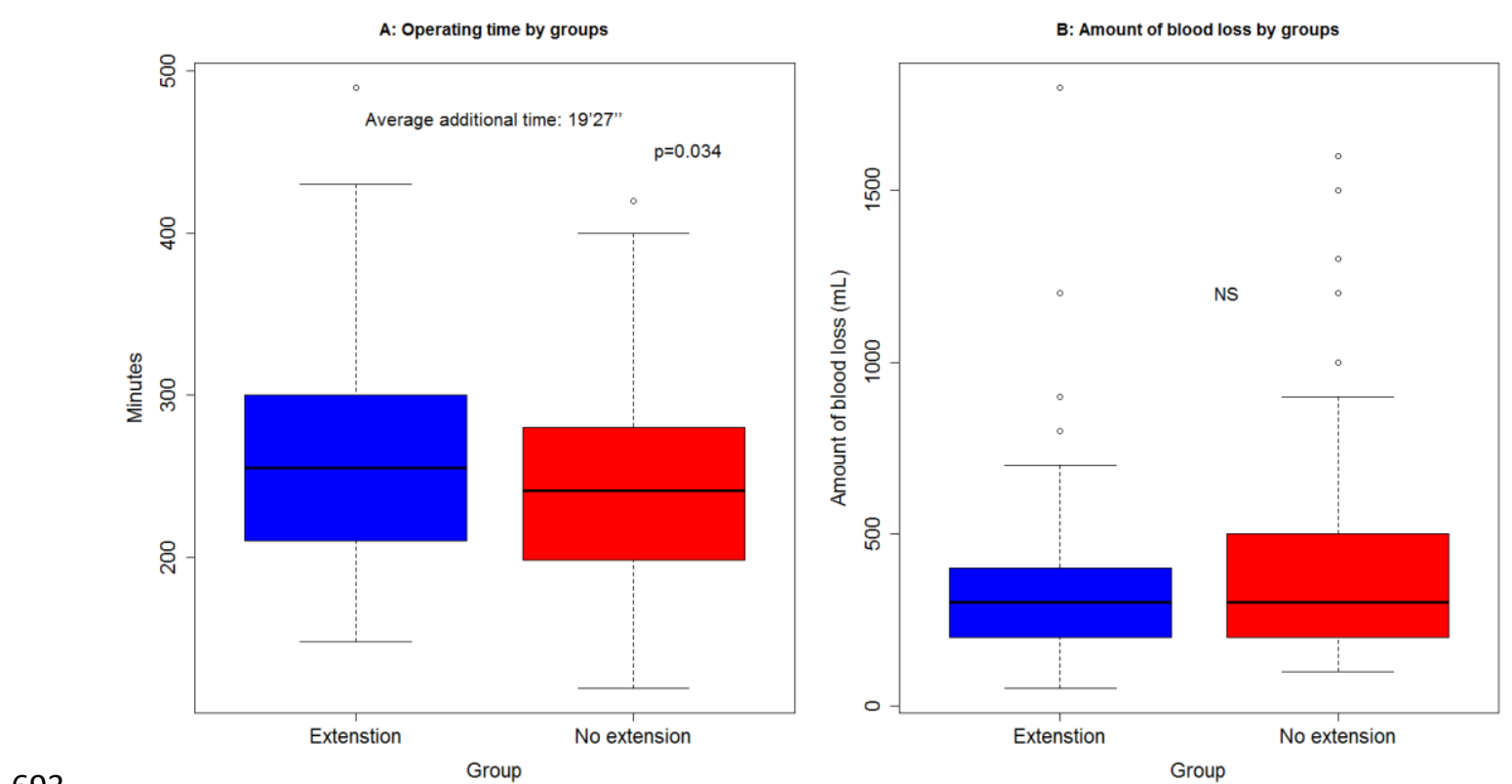

694 

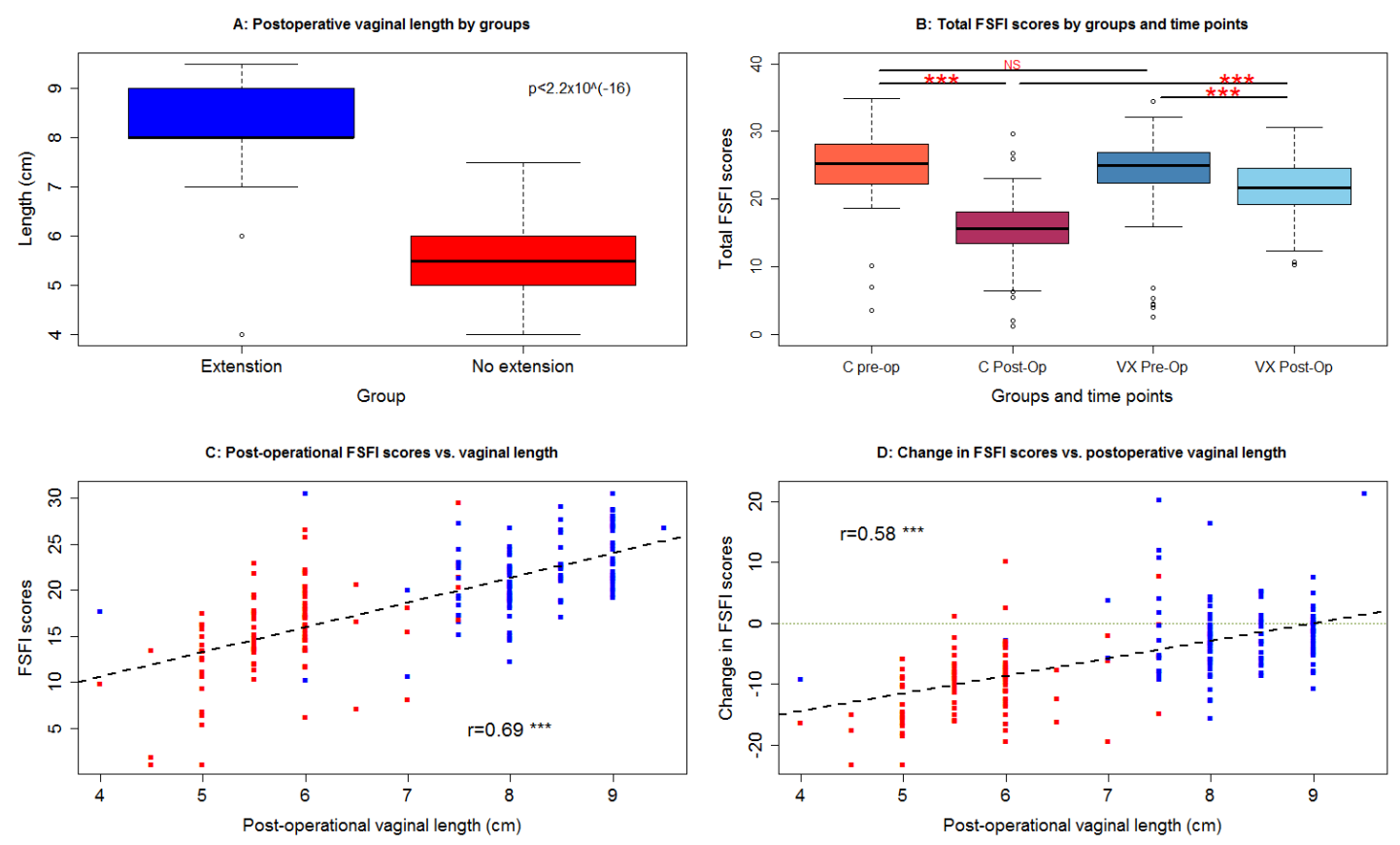

695 


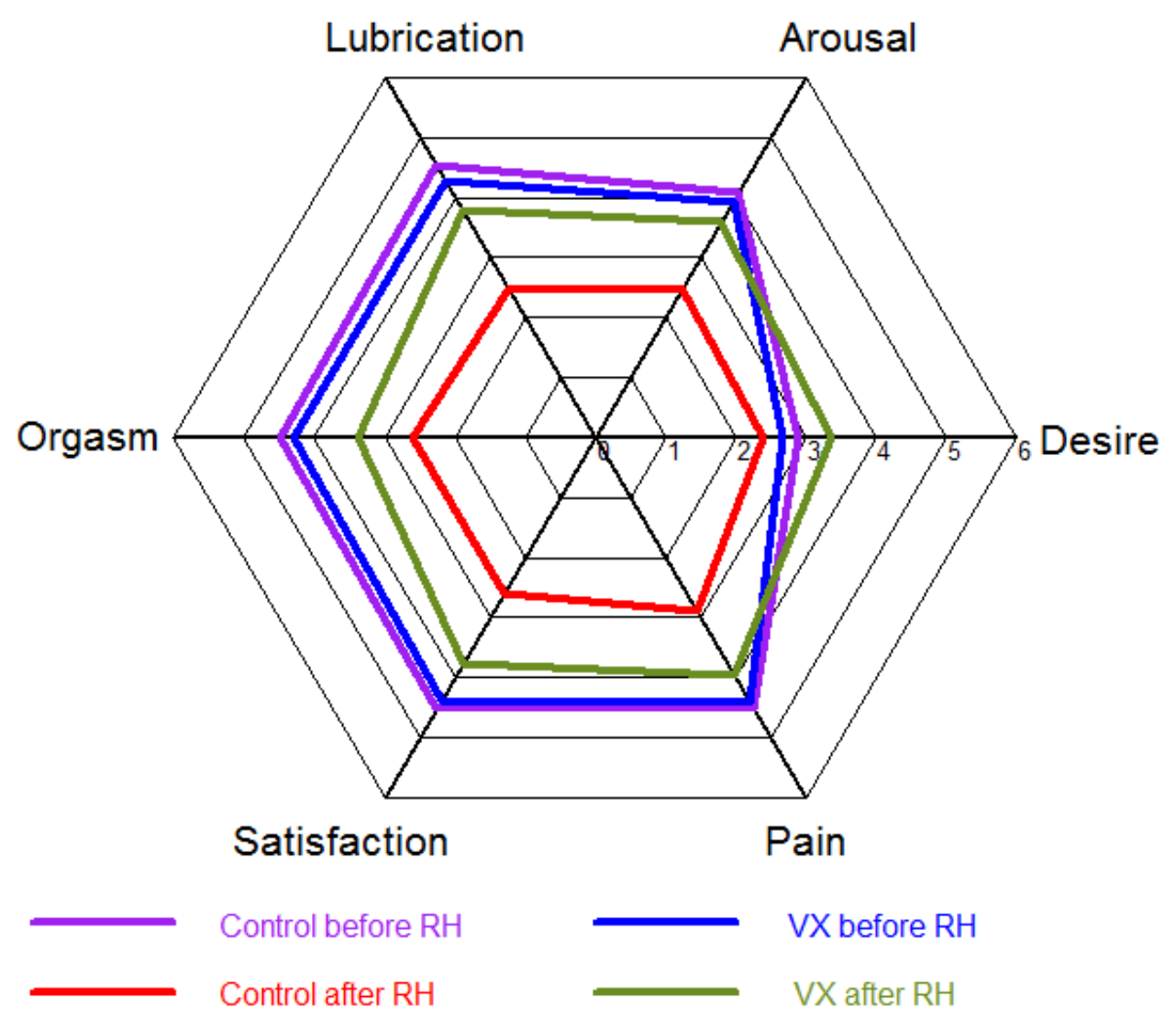

697

698 
Possible ways to improve sexual function for cervical cancer survivors following radical hysterectomy

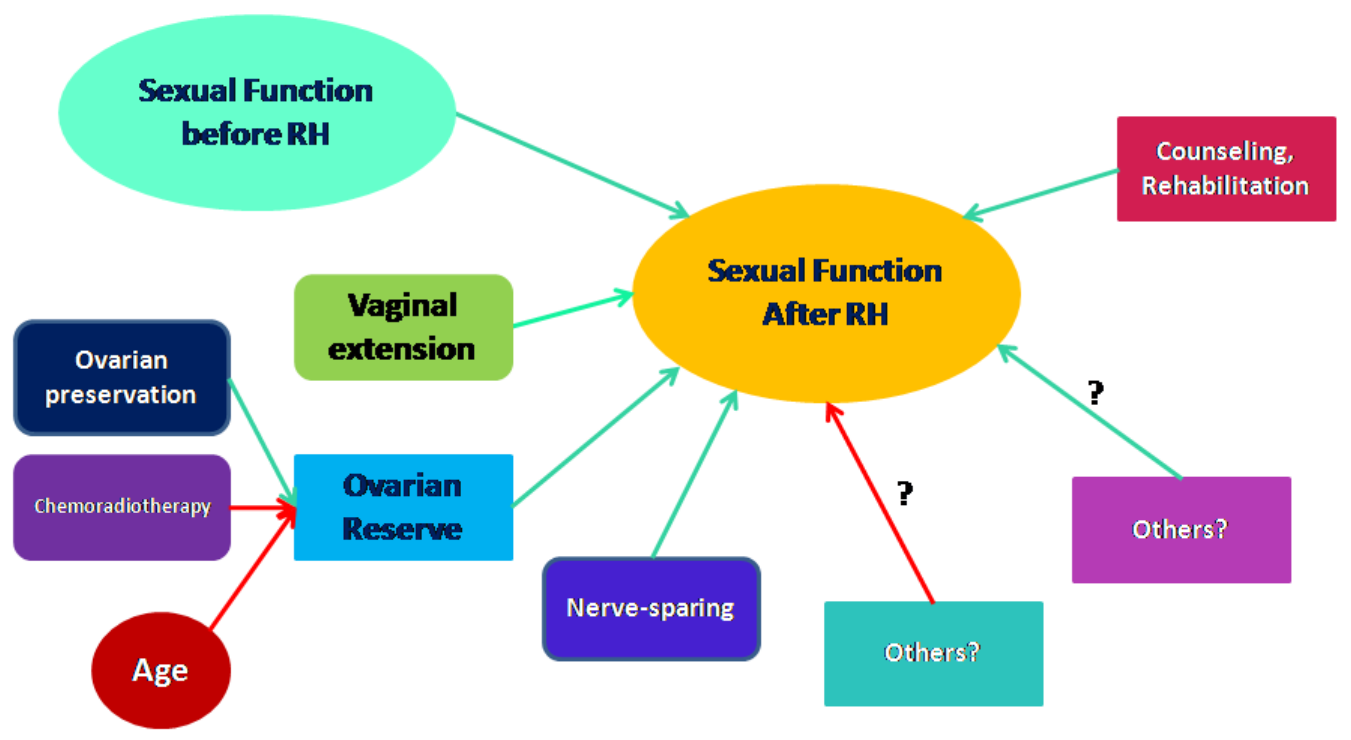

699 


\begin{tabular}{|c|c|c|c|}
\hline Variable & $\begin{array}{l}\text { Conventional RH } \\
\text { group }(\mathbf{C}) \\
(\mathbf{n}=101)\end{array}$ & $\begin{array}{l}\text { Vaginal extension } \\
\text { following } R H \\
\text { group }(\mathrm{VX}) \\
(\mathrm{n}=115) \\
\end{array}$ & $\begin{array}{l}\text { p-value of the } \\
\text { difference }\end{array}$ \\
\hline Age (years) & $\begin{array}{l}38.3 \pm 4.0 \\
39(32-45)\end{array}$ & $\begin{array}{l}36.0 \pm 4.4 \\
36(33-39)\end{array}$ & 0.0002 \\
\hline $\begin{array}{r}\text { Parity } \\
\qquad \begin{array}{r}0 \\
1 \\
2 \\
\geqslant 3\end{array}\end{array}$ & $\begin{array}{c}4(4.0 \%) \\
70(69.3 \%) \\
23(22.8 \%) \\
4(4.0 \%) \\
\end{array}$ & $\begin{array}{c}13(11.3 \%) \\
74(64.3 \%) \\
23(20.0 \%) \\
5(4.3 \%) \\
\end{array}$ & 0.25 \\
\hline $\begin{array}{l}\text { FIGO stage } \\
\text { Ia1 with positive margin } \\
\text { Ia2 } \\
\text { Ib1 } \\
\text { Ib2 } \\
\text { IIa1 } \\
\text { IIa2 }\end{array}$ & $\begin{array}{c}4(4.0 \%) \\
3(3.0 \%) \\
69(68.3 \%) \\
9(8.9 \%) \\
5(5.0 \%) \\
11(10.9 \%) \\
\end{array}$ & $\begin{array}{c}10(8.7 \%) \\
2(1.7 \%) \\
79(68.7 \%) \\
8(7.0 \%) \\
9(7.8 \%) \\
7(6.1 \%) \\
\end{array}$ & 0.47 \\
\hline $\begin{array}{l}\text { Histologic subtype } \\
\text { Squamous carcinoma } \\
\text { Adenocarcinoma } \\
\text { Adenosquamous carcinoma } \\
\text { Neuroendocrine cell } \\
\text { carcinoma }\end{array}$ & $\begin{array}{c}82(81.2 \%) \\
12(11.9 \%) \\
6(5.9 \%) \\
1(1.0 \%)\end{array}$ & $\begin{array}{c}97(84.3 \%) \\
12(10.4 \%) \\
5(4.3 \%) \\
1(0.9 \%)\end{array}$ & 0.92 \\
\hline Depth of invasion (mm) & $2.35 \pm 0.52$ & $2.39 \pm 0.51$ & 0.55 \\
\hline $\begin{array}{l}\text { Involvement of vaginal fornix } \\
\qquad e s \\
\text { No }\end{array}$ & $\begin{array}{l}11(10.9 \%) \\
90(89.1 \%) \\
\end{array}$ & $\begin{array}{r}13(11.3 \%) \\
102(88.7 \%) \\
\end{array}$ & 1.0 \\
\hline $\begin{array}{c}\text { Parametrial involvement } \\
\text { Yes } \\
\mathrm{No} \\
\end{array}$ & $\begin{array}{c}1(1.0 \%) \\
100(99.0 \%)\end{array}$ & $\begin{array}{r}2(1.7 \%) \\
113(98.3 \%) \\
\end{array}$ & 1.0 \\
\hline $\begin{array}{c}\text { Involvement of vaginal wall } \\
\text { Yes } \\
\text { No }\end{array}$ & $\begin{array}{c}0(0.0 \%) \\
101(100.0 \%)\end{array}$ & $\begin{array}{c}5(4.3 \%) \\
110(95.7 \%)\end{array}$ & 0.062 \\
\hline $\begin{array}{c}\text { Lymphatic/vascular space } \\
\text { invasion (LVSI) } \\
\text { Yes } \\
\text { No }\end{array}$ & $\begin{array}{l}19(18.8 \%) \\
82(81.2 \%)\end{array}$ & $\begin{array}{l}30(26.1 \%) \\
85(73.9 \%)\end{array}$ & 0.25 \\
\hline
\end{tabular}




\begin{tabular}{|c|c|c|c|}
\hline $\begin{array}{c}\text { Parametrial metastasis } \\
\text { Yes } \\
\mathrm{No}\end{array}$ & $\begin{array}{c}1(1.0 \%) \\
100(99.0 \%)\end{array}$ & $\begin{array}{c}3(2.6 \%) \\
112(97.4 \%)\end{array}$ & 0.62 \\
\hline $\begin{array}{l}\text { Pelvic lymphnode } \\
\text { involvement } \\
\text { Yes } \\
\mathrm{No}\end{array}$ & $\begin{array}{c}6(5.9 \%) \\
95(94.1 \%)\end{array}$ & $\begin{array}{c}11(9.6 \%) \\
104(90.4 \%)\end{array}$ & 0.45 \\
\hline $\begin{array}{c}\text { Ovary preservation procedure } \\
\text { Yes } \\
\text { No }\end{array}$ & $\begin{array}{l}90(89.1 \%) \\
11(10.9 \%)\end{array}$ & $\begin{array}{c}105(91.3 \%) \\
10(8.7 \%)\end{array}$ & 0.65 \\
\hline Operating time (min) & $\begin{array}{l}242.3 \pm 64.7 \\
241(198--290)\end{array}$ & $\begin{array}{l}261.7 \pm 62.6 \\
255(210-300)\end{array}$ & 0.034 \\
\hline Amount of blood loss (mL) & $\begin{array}{l}373.2 \pm 285.3 \\
300(200-500)\end{array}$ & $\begin{array}{l}360.3 \pm 243.8 \\
300(200-400)\end{array}$ & 0.80 \\
\hline $\begin{array}{l}\text { Length of resected vaginal } \\
\text { wall }(\mathrm{cm}) \\
\text { At } 12 \text { o'clock } \\
\text { At } 3 \text { o'clock } \\
\text { At } 6 \text { o'clock } \\
\text { At } 9 \text { o'clock }\end{array}$ & $\begin{array}{l}2.76 \pm 0.86 \\
2.97 \pm 0.84 \\
3.18 \pm 0.89 \\
2.86 \pm 0.88 \\
\end{array}$ & $\begin{array}{l}2.85 \pm 0.95 \\
2.93 \pm 0.73 \\
3.33 \pm 0.92 \\
2.86 \pm 0.77 \\
\end{array}$ & $\begin{array}{l}0.44 \\
0.95 \\
0.18 \\
0.96\end{array}$ \\
\hline $\begin{array}{l}\text { Cases with intra-operative } \\
\text { complication (ureteral injury) }\end{array}$ & $4(4.0 \%)$ & $2(1.7 \%)$ & 0.42 \\
\hline $\begin{array}{c}\text { Ovarian preservation } \\
\text { Yes } \\
\mathrm{No} \\
\end{array}$ & $\begin{array}{l}90(90.1 \%) \\
11(10.9 \%) \\
\end{array}$ & $\begin{array}{c}105(91.3 \%) \\
10(8.7 \%) \\
\end{array}$ & 0.65 \\
\hline Length of hospital stay (day) & $\begin{array}{l}11.1 \pm 5.5 \\
10(3--27) \\
\end{array}$ & $\begin{array}{l}9.7 \pm 4.0 \\
9(3-26) \\
\end{array}$ & 0.13 \\
\hline $\begin{array}{l}\text { Time to first passing of flatus } \\
\text { (day) }\end{array}$ & $2.10 \pm 0.36$ & $2.19 \pm 0.46$ & 0.071 \\
\hline $\begin{array}{l}\text { Postoperative adjuvant } \\
\text { therapy } \\
\text { None } \\
\text { Chemotherapy only } \\
\text { Radiotherapy only } \\
\text { Chemoradiotherapy } \\
\text { Missing }\end{array}$ & $\begin{array}{c}50(49.5 \%) \\
4(4.0 \%) \\
2(2.0 \%) \\
45(44.6 \%) \\
\quad 0\end{array}$ & $\begin{aligned} & 52(45.6 \%) \\
& 3(2.6 \%) \\
& 5(4.4 \%) \\
& 54(47.4 \%) \\
& 1\end{aligned}$ & 0.70 \\
\hline $\begin{array}{l}\text { Number of deaths by the last } \\
\text { follow-up }\end{array}$ & $2(2.0 \%)$ & $2(1.7 \%)$ & 1.00 \\
\hline
\end{tabular}


Table 2. Sexual functions before and 1-year after surgery.

\begin{tabular}{|c|c|c|c|}
\hline Item & $\begin{array}{l}\text { Conventional RH } \\
\text { group }(\mathbf{C}) \\
(\mathbf{n}=\mathbf{1 0 1})\end{array}$ & $\begin{array}{l}\text { Vaginal extension } \\
\text { following } \\
\text { group }(\mathrm{VX}) \\
(\mathrm{n}=115)\end{array}$ & $\begin{array}{l}\text { p-value of the } \\
\text { difference }\end{array}$ \\
\hline Pre-operative FSFI & & & \\
\hline Desire & $2.90 \pm 1.08$ & $2.66 \pm 0.98$ & 0.12 \\
\hline Arousal & $4.09 \pm 0.99$ & $3.93 \pm 1.16$ & 0.61 \\
\hline Lubrication & $4.52 \pm 0.97$ & $4.27 \pm 1.21$ & 0.13 \\
\hline Orgasm & $4.49 \pm 0.93$ & $4.28 \pm 1.20$ & 0.29 \\
\hline Satisfaction & $4.51 \pm 0.80$ & $4.38 \pm 0.86$ & 0.31 \\
\hline Pain & $4.50 \pm 1.13$ & $4.40 \pm 1.28$ & 0.77 \\
\hline Total score & $25.0 \pm 4.8$ & $23.9 \pm 5.4$ & 0.23 \\
\hline $\begin{array}{l}\text { Number of missing } \\
\text { FSFI score due to } \\
\text { divorce or the lack of } \\
\text { sexual activity } \\
\text { postoperationally }\end{array}$ & $11(10.9 \%)$ & $7(6.1 \%)$ & 0.23 \\
\hline $\begin{array}{l}\text { Length of vagina } \\
\text { measured } 1 \text { year after } \\
\text { surgery }(\mathrm{cm})\end{array}$ & $\begin{array}{l}5.68 \pm 0.71 \\
5.5(5-6)\end{array}$ & $\begin{array}{l}8.19 \pm 0.80 \\
8(8-9)\end{array}$ & $<2.2 \times 10^{-16}$ \\
\hline $\begin{array}{l}\text { Circulating FSH level } \\
(\mathrm{mIU} / \mathrm{mL}) \\
\text { \# of missing }\end{array}$ & $\begin{array}{l}32.1 \pm 35.8 \\
11.0(4.1-135.2) \\
24 \\
\end{array}$ & $\begin{array}{l}29.7(39.5) \\
8.3(2.1-146.5) \\
14\end{array}$ & 0.026 \\
\hline $\begin{array}{l}\text { Circulating LH level } \\
(\mathrm{mIU} / \mathrm{mL}) \\
\text { \# of missing }\end{array}$ & $\begin{array}{l}22.7 \pm 23.8 \\
9.8(2.2-87.9) \\
\quad 24 \\
\end{array}$ & $\begin{array}{l}19.7 \pm 24.0 \\
8.2(1.9-126.1) \\
14\end{array}$ & 0.13 \\
\hline 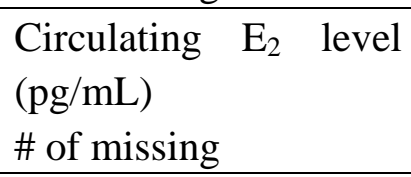 & $\begin{array}{l}67.5 \pm 74.3 \\
55.0(5.0-552.0) \\
24 \\
\end{array}$ & $\begin{array}{c}85.6 \pm 71.9 \\
78.0(4.0-337.0) \\
14 \\
\end{array}$ & 0.046 \\
\hline $\begin{array}{lll}\begin{array}{l}\text { Circulating } \\
(\mathrm{ng} / \mathrm{mL})\end{array} & & \text { level } \\
\text { \# of missing } & & \\
\end{array}$ & $\begin{array}{l}3.7 \pm 5.5 \\
0.9(0.01-32.0) \\
27 \\
\end{array}$ & $\begin{array}{l}4.3 \pm 6.0 \\
1.6(0.01-25.5) \\
14\end{array}$ & 0.22 \\
\hline $\begin{array}{l}\text { Time to start having } \\
\text { sexual intercourse } \\
\text { after surgery (in } \\
\text { month) }\end{array}$ & $\begin{array}{l}5.86 \pm 1.49 \\
6(5-7)\end{array}$ & $\begin{array}{l}5.19 \pm 1.67 \\
5(4-6)\end{array}$ & 0.0001 \\
\hline Post-operative FSFI & & & \\
\hline Desire & $2.39 \pm 0.75^{* * *}$ & $3.36 \pm 0.74 * * *$ & $1.3 \times 10^{-14}$ \\
\hline Arousal & $2.48 \pm 0.91 * * *$ & $3.59 \pm 0.87 * * *$ & $3.6 \times 10^{-16}$ \\
\hline Lubrication & $2.46 \pm 1.06 * * *$ & $3.78 \pm 0.97 * * *$ & $4.3 \times 10^{-16}$ \\
\hline Orgasm & $2.61 \pm 1.19 * * *$ & $3.37 \pm 0.82 * * *$ & $6.5 \times 10^{-7}$ \\
\hline Satisfaction & $2.59 \pm 1.12 * * *$ & $3.75 \pm 0.95^{* * *}$ & $4.1 \times 10^{-13}$ \\
\hline
\end{tabular}




\begin{tabular}{|c|c|c|c|}
\hline Pain & $2.90 \pm 1.34 * * *$ & $3.95 \pm 0.84 * * *$ & $6.8 \times 10^{-10}$ \\
Total score & $15.4 \pm 5.2 * * *$ & $21.8 \pm 4.12 * * *$ & $<2.2 \times 10^{-16}$ \\
\# missing & 13 & 7 & \\
\hline
\end{tabular}

713

$714 * * *$ : Statistical significance level $\mathrm{p}<0.001$ as compared with the baseline levels.

715

716

717 